\title{
Morbidity and Mortality among Patients Referred from District Hospitals to Butare University Teaching Hospital Chub/Obgyn Department in Post-Partum and Post-Operative Period
}

\author{
E. Habimana ${ }^{*}$, J. B. Kalibushi ${ }^{2}$, J. Ndoli ${ }^{3}$ and C. Bayingana ${ }^{4}$ \\ ${ }^{1}$ Senior Resident in Obstetrics and Gynecology department, Butare University \\ Teaching Hospital (CHUB), Rwanda \\ ${ }^{2}$ Head of Obstetrics and Gynecology department, Butare University \\ Teaching Hospital (CHUB), Rwanda \\ ${ }^{3}$ Medical Director, Butare University Teaching Hospital (CHUB), Rwanda \\ ${ }^{4}$ Head of Clinical Laboratory, Butare University Teaching Hospital (CHUB), Rwanda \\ *Corresponding author
}

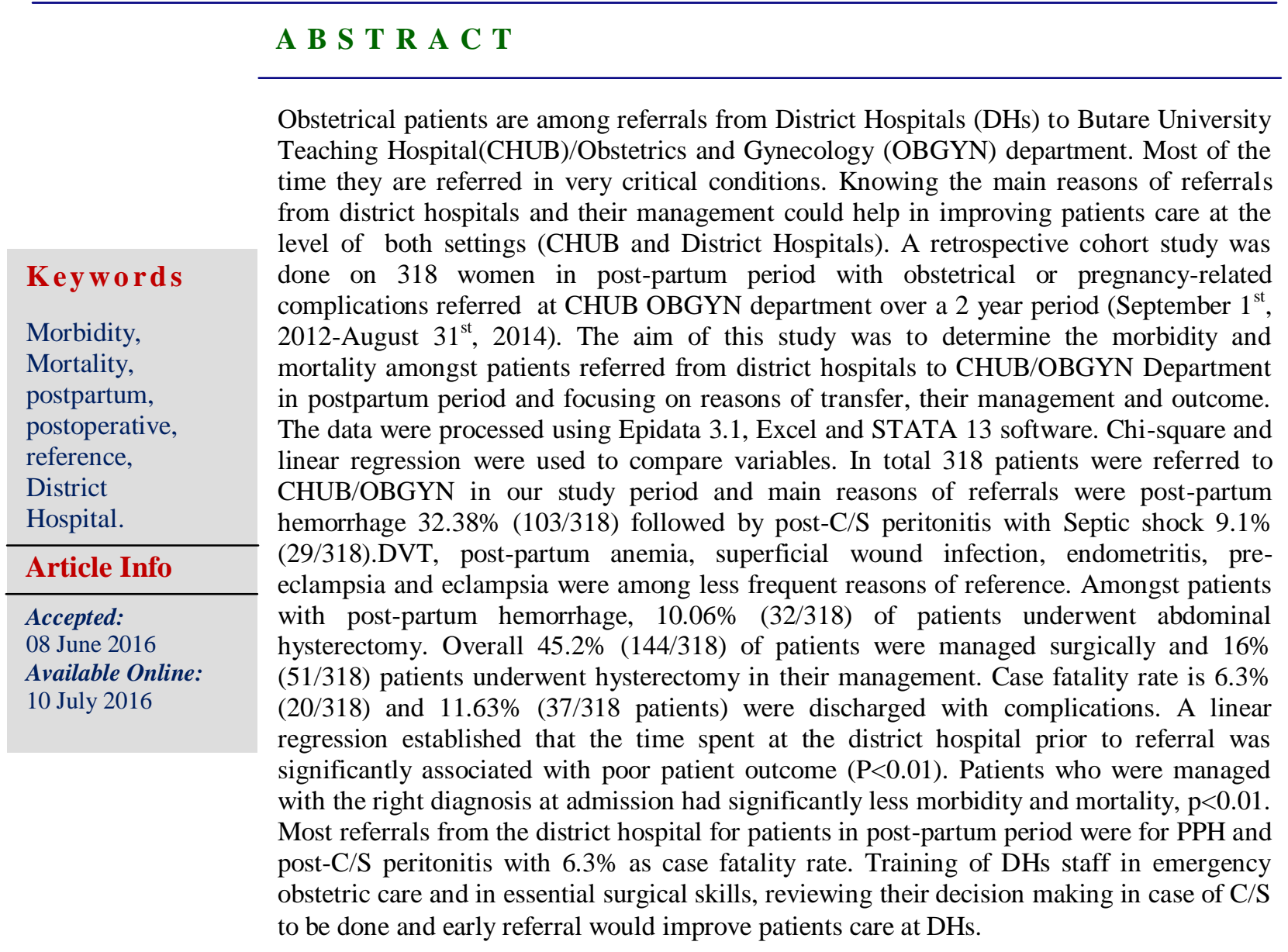




\section{Introduction}

Butare University Teaching Hospital (CHUB) as a referral hospital receives patients from district hospitals mainly from Southern and Western Provinces in Rwanda. Health care providers at district hospitals refer patients either because they lack skills, facilities, or both to manage a given clinical condition (Dean et al., 2006). This study evaluates referrals in the postpartum and in post-operative period. As it is known, the burden of maternal ill-health includes not only the levels of maternal mortality and complications during pregnancy and around the time of delivery but also extends to the standard postpartum period of 42 days with consequences of obstetric complications and poor management at delivery (Mavalankar et al., 2005; WHO/UNICEF/UNFPA/World Bank, 2012). Globally, the total number of maternal deaths decreased by from 543000 in 1990 to 287000 in 2010. Likewise, the global maternal mortality ratio (MMR) declined from 400 maternal deaths per 100 000 live births in 1990 to 210 in 2010, representing an average annual decline of 3.1 per cent (Ferdous et al., 2012).

Throughout history, pregnancy has carried a high risk of death secondary to complications as obstructed labor, ruptured uterus, postpartum hemorrhage, postpartum infection, hypertensive disease of pregnancy, and complications stemming from unsafe abortion. Maternal mortality is still high as about 800 women die from pregnancy- or childbirth-related complications around the world every day and in 2010, 287000 women died during and following pregnancy and childbirth. Almost all of these deaths occurred in lowresource settings, and most could have been prevented (Conde-Agudelo et al., 2004; WHO, 2012). Improving maternal health is one of the eight Millennium Development
Goals (MDGs) adopted by the international community in 2000. Under MDG5, countries committed to reducing maternal mortality by three quarters between 1990 and 2015. Since 1990, maternal deaths worldwide have dropped by $47 \%$. In subSaharan Africa, a number of countries have halved their levels of maternal mortality since 1990. In other regions, including Asia and North Africa, even greater headway has been made. However, between 1990 and 2010, the global maternal mortality ratio (i.e. the number of maternal deaths per 100 000 live births) declined by only $3.1 \%$ per year. This is far from the annual decline of $5.5 \%$ required to achieve MDG5. Women die as a result of complications during and following pregnancy and childbirth. Most of these complications develop during pregnancy. Other complications may exist before pregnancy but are worsened during pregnancy. The major complications that account for $80 \%$ of all maternal deaths are: severe bleeding (mostly bleeding after childbirth), infections (usually after childbirth), high blood pressure during pregnancy (pre-eclampsia and eclampsia), unsafe abortion. The remainder are caused by or associated with diseases such as malaria, and AIDS during pregnancy (Conde-Agudelo et al., 2004; WHO, 2012).

In Rwanda, a comparison of maternal mortality ratios from 2000,2005,2010 and 2014-2015 Rwanda Demographic and Health Surveys (RDHS) revealed that maternal mortality has been continuously decreasing overtime from 1071,750,476and 210deaths per 100,000 livebirths respectively (National institute of statistics, Rwanda., Ministry of Finance and Economic Planning, Rwanda., Ministry of Health, Rwanda, 2016).

Knowing the main reasons of referrals from district hospitals and their management 
could help in improving patients care at the level of both settings (CHUB and District Hospitals). The hypothesis was that there was a high mortality rate amongst patients referred from District Hospitals to CHUB/OBGYN Department. Early diagnosis and early transfer will decrease this mortality rate.

The aim of this study was to determine the morbidity and mortality amongst patients referred from district hospitals to CHUB/OBGYN Department in postpartum period and focusing on reasons of transfer, their management and outcome.

\section{Materials and Methods}

This is a retrospective cohort study of patients referred post-partum from district hospitals to CHUB/OBGYN department in a period of two years (September 1st, 2012 to August 31st, 2014). This cohort included all patients referred from district hospitals to CHUB/OBGYN department within 42 days of delivery regardless of mode of delivery and includes patients who were at least 24 weeks gestational age prior to delivery. Patients referred from district hospitals for gynecologic indications, all patients who underwent spontaneous or therapeutic abortion, all patients referred who are not in postpartum period and all patients who delivered at CHUB were excluded. All files for women in the cohort were reviewed and de identified data were extracted using a preset data collection form. The data were processed using Epidata 3.1, Excel and STATA 13 software. Data were doublechecked before analysis. Chi-square and linear regression were used to compare variables. $\quad \mathrm{P}<0.05$ was considered significant and power was $80 \%$. The proposal protocol was approved by CHUB Research Committee and permission from CHUB authorities was obtained before starting data collection.
To ensure confidentiality of data confined in patient files, the data extracted did not contain patient identifiers such as name, medical record number, date of birth, or address. Data extraction was conducted by a trained health professional (a midwife, an intern, medical student) who are trained on medical research ethics.

Our main objective was to determine the morbidity and mortality amongst patients referred from district hospitals to CHUB/OBGYN Department in postpartum period. We calculated the mortality of women referred within 42 days postpartum as well as the percentage of women who had complications including surgical site infection and repeat laparotomy.

\section{Results and Discussion}

In a period of two years, three hundred eighteen (318) patients fitting in our criteria were admitted and managed at CHUB/OBGYN department from District Hospitals (DHs). There were from 15 district hospitals and ranged from early to late post-partum period. The mode of delivery was either Cesarian-Section or vaginal delivery. Their mean age is 29.6years, and the youngest patient is 16 years and the oldest patient is 65 years. Most of them were of 25-34 year-old $49.37 \%$ (157/318). Most of the patients referred to CHUB from DHs were of 1 to 3 parities (64.8\%), $48.7 \%$ of all referred patients were delivered eutocically one to three times, $39.6 \%$ reported one prior cesarian delivery, while a majority of referred patients do not have any history of C/Section (49.1\%), $33.3 \%$ of recently born neonates did not survive. The prevalence of HIV among referred patients were $3.1 \% \quad(10 / 318$ patients) and $0.6 \%$ of them were taking only Bactrim while $2.5 \%$ were taking HAART (Highly Active Anti-Retroviral 
Treatment).The majority of referred patients (89.3\%) were with no history of chronic disease while $7.5 \%$ were having asthma in their history.(Table 1)

We found the majority of referrals were for PPH (32.38\% (103/318)) either post-C/S or post-NSVD, a majority of these patients came in at CHUB in hemorrhagic shock. Septic shock, post-C/S peritonitis, DVT, post-partum anemia, surperficial wound infection after $\mathrm{C} / \mathrm{S}$, Endometritis, Preeclampsia and Eclampsia were also among the main reasons of referral. (Table 3)

Among 103 patients referred for mainly $\mathrm{PPH}, 12.6 \%$ Of them (13/103) were managed conservatively, $11.6 \%$ patients (12/103) were managed only medically and more than a half of patients with PPH 56.3\% (58/103) were managed both medically and surgically. 32 patients $(10 \%$ of the total referred patients) underwent abdominal hysterectomy for hemostasis. Patients who were diagnosed to have Post-C/S peritonitis with other associated conditions like septic shock were $9.1 \%$ patients (29/318) and they were mainly managed medically and surgically together. 25patients $25 / 318=7.8 \%$ ) underwent laparotomy and 5 patients $(5 / 318=1.57 \%$ of the referred patients) of them underwent hysterectomy as the uterus were necrotised and impossible to conserve.

$6.2 \%$ Patients (20/318) were diagnosed to have superficial surgical site wound infection and only 4 patients $(3 / 20=20 \%)$ had hysterectomy included in their management. 5.34\% patients (17/318) were diagnosed to have pre-eclampsia or eclampsia and were managed accordingly. Overall $45.2 \%(144 / 318)$ of all referred patients to CHUB/OBYGN in post-partum period in study period were managed surgically and $16 \%(51 / 318)$ patients underwent hysterectomy among other management (Table 4).

This study showed that 6.3\% (20/318) patients referred to $\mathrm{CHUB} / \mathrm{OBGYN}$ died and most of them $65 \%(13 / 20)$ patients died within 3 days of admission, $40 \%$ (8/20) died in ICU. $80.20 \%$ (255/318) patients improved and were discharged and most of them $40.8 \%(104 / 255)$ were discharged within ten days of admission and only $16.5 \%$ (42/255) patients of them spent more than 20days at the hospital. In total $11.63 \% \quad(37 / 318$ patients) were discharged with complications and 1.9\%patients (6/318) were referred to another hospital mainly at KIGALI University Teaching Hospital (CHUK) and at Rwanda Military Hospitals, primarily for urologist review.

$72.3 \%$ of all referred patients to CHUB /OBGYN were managed with the right diagnosis from their admission. $3.5 \%$ (11/318) patients of referred patients to CHUB/OBGYN had various kinds of fistulas, $1.9 \%$ had ureteric or bladder injury as post-partum complications and the majority of patients recovered with no complication. In total $8.2 \%$ patients $(26 / 318)$ were admitted in ICU and of them $34.6 \%$ $(9 / 26)$ patients spent there more than six days.

Among patients who were diagnosed with PPH at CHUB and managed accordingly, $44.6 \%$ (46/103) had different kinds of complications, $5.8 \% \quad(6 / 103)$ patients developed fistulas, primarily vesico-vaginal fistulas.

Among patients who were diagnosed to have post-C/S peritonitis, $17.2 \% \quad(5 / 29)$ had fistulas, mainly enterocutaneous fistulas and $6.8 \%(2 / 29)$ had bowel injury with colostomy or ileostomy plus fistula. (Table 6) 
When we analysed health conditions at discharge day and admission on ICU, we found that there was a significant association. Those patients who were admitted in ICU were also prone to have complications and the risk of death. $(\mathrm{P}=0.04)$. Results of this study also showed that patients from Western province district hospitals mainly MIBIRIZI and GIHUNDWE DHs spent more than 16hours at the hospital before being referred (Table 7). A linear regression established that the time spent to DHs before being referred could statistically significantly predict complications that patients had with their health conditions at discharge day, $\mathrm{P}=0.0005$, more the patient spent more time at $\mathrm{DH}$ the more she got severe complications and the more she is likely to die if she has post-c/section peritonitis $(\mathrm{P}=0.007)$ and Patients who were managed with the right diagnosis at admission were less likely to have complications and to die, , $\mathrm{P}=0.0033$. $50 \%$ patients $(11 / 20)$ who died spent between 0 and 6hours at their respective DHs before being referred and there were having PPH as the working diagnosis. (Table 6)

In this study, when the effect of other chronic diseases on the conditions of patients all along in their admission time was studied, we found that most of patients had no significant medical history and most were HIV negative. No significant association between past medical history and the outcomes evaluated in this study $(\mathrm{P}=0.9)$

Most of patients $60 \%(12 / 20)$ who died were HIV negative, there was no association between having HIV and the risk of death during the hospitalization. No patient with HIV had complications or referred to another hospital. $(\mathrm{P}=0.4)$. In a period of two years, three hundred eighteen (318) patients were admitted and managed at
CHUB/OBGYN department from District Hospitals (DHs) for obstetrical or pregnancy-related complications between the early and late post-partum period.

DHs from Western Province had roughly a half of transfers to CHUB during our study period (MIBIRIZI DH: 20.8\%, BUSHENGE DH: $\quad 7.2 \%$, KIBOGORA: $6.6 \%$, GIHUNDWE: $11.6 \%$ ), and we cannot know exactly why their patients are having complications. Experience and surgical skills of doctors in those hospitals can be assessed with other conditions like facilities, nursing staffs, materials and so on (WHO, 2012).

This study showed that almost a third $32.38 \%$ (103/318) of patients referred had PPH as morbidity associated with other conditions like hypovolemic shock, anemia, etc ; followed by Septic shock and post-C/S peritonitis, deep venous thrombosis (DVT), post-partum anemia, surperficial wound infection after $\mathrm{C} / \mathrm{S}$, Endometritis, Preeclampsia and Eclampsia. Our results are not far from those of other results that show that women suffer pregnancy-related complications before, during and even after delivery and among post-partum complications include PPH, Post-partum infection, pre-eclampsia and eclampsia with DVT (Ferdous et al., 2012; Conde-Agudelo et al., 2004; WHO, 2012; Iyengar et al., 2012). In a study done in Tanzania on maternal near miss (MNM) showed that major causes were eclampsia and postpartum haemorrhage and CS complications accounted for $7.9 \%$ of the MNM events and $13 \%$ of the maternal deaths (Litorp et al., 2014). Another study done at three university hospitals with a high rate of CS in Tehran-Iran on Maternal near miss revealed that Severe postpartum hemorrhage $(35 \%, 29 / 82)$, severe preeclampsia $(32 \%, 26 / 82)$, and placenta previa/abnormally invasive placenta $(10 \%$, 
8/82) were the most frequent causes of MNM (Mohammadi et al., 2016). Rulisa et $a l$ in their study on Maternal near miss and mortality in a tertiary care hospital in Rwanda revealed that the majority of severe obstetric morbidity and mortalities resulted from: sepsis/peritonitis (30.2\%)--primarily following caesarean deliveries, hypertensive disease $(28.6 \%)$, and hemorrhage $(19.3 \%)$ (Mantel et al., 1998).

When we looked at the management of patient diagnosed to have $\mathrm{PPH}$, this study revealed that among 103 patients referred for mainly PPH, $12.6 \%$ of them (13/103) were managed conservatively, $11.6 \%$ patients (12/103) were managed only medically and more than a half of patients with PPH 56.3\% (58/103) were managed both medically and surgically. And among them 32 patients $32 / 318=10.06 \%$ ) underwent abdominal hysterectomy for hemostasis that equals to $10.06 \%$ of the total referred patients. The above management is comparable to the management of such patients from other studies as they mention that the first step is to attempt conservative management by giving uterotonics like oxytocin, cytotec, ergot alkaloids such ergometrine or its derivative methylergonovine (methylergometrine, methergine), if conservative management fails invasive treatment of $\mathrm{PPH}$ is initiated to avoid severe morbidity and mortality ; uterine balloon tamponade, uterine compression sutures, angiographic arterial embolisation, uterine ligation and hysterectomy are being used in this case. However Bassey et al, in their study on emergency peripartum hysterectomy in a low resource setting found that the commonest indication of peripartum hysterectomy was uterine rupture but for us hysterectomy was done when uterus was damaged either through fetal extraction and unable to repair or when there is atonic uterine that is responding to conservative and invasive procedure rather than hysterectomy (Rwanda et al., 2009; Litorp et al., 2014).

Results of this study highlight the big number of mother who lost their uterus and so their obstetrical future. In total $45.2 \%$ (144/318) of all referred patients to CHUB/OBYGN in post-partum period in study period were managed surgically and $16 \% \quad(51 / 318)$ patients underwent hysterectomy among other management. when we look at the management of referred patients with post-partum infection, $9.1 \%$ patients (29/318) had post-C/S peritonitis associated with other conditions like septic shock. 5 patients $(5 / 29=17.24 \%$ or $5 / 318=$ $1.57 \%)$ of them plus 4 patients $(3 / 20=20 \%)$ among patients diagnosed initially with superficial site wound infection underwent hysterectomy as the uterus were necrotised and impossible to conserve. Recent cesarian section was associated with more PPH and more post-partum infection compared to vaginal delivery and they were more likely to undergo hysterectomy $(\mathrm{P}=0.021)$. Those results are similar so those of other studies where pueriperal infection and post$\mathrm{c} /$ section infection either superficial surgical site infection and post-CS peritonitis are among severe morbidity and causes of maternal near miss and mortality. Severe infection ends up with sepsis and septic shock and most patients with sepsis post-CS end up with having their uterus removed as it is necrotised and impossible to conserve.

Patients from Western province district hospitals mainly MIBIRIZI and GIHUNDWE DHs spent more than 16hours at the hospital before being referred. And a linear regression established that the time spent to DHs before being referred could statistically significantly predict complications that patients had with their 
health conditions at discharge day. Results of this study showed that the more the patient spent more time at $\mathrm{DH}$ the more she got severe complications and the more she is likely to die if she has post-c/section peritonitis $(\mathrm{P}=0.007)$ and Patients who were managed with the right diagnosis at admission were less likely to have complications and to die, $\mathrm{P}<0.01$. $50 \%$ patients (11/20) who died spent at least 6hours at their respective DHs before being referred and there were having $\mathrm{PPH}$ as the working diagnosis, this is too much time as we know that PPH can kill a patient within two hours post-partum if nothing is done. When we add this delay to the long way from Western province DHs and to the questionable availability of obstetrical emergency, time interval between the admission, diagnosis and starting management where with an average time of 72 hours we understand that most of patients with PPH could not even arrive at CHUB. Time matters in case of emergency obstetrical care.

Table.1 Characteristics

\begin{tabular}{|c|c|c|}
\hline Age group & Number & Percent \\
\hline $16-24$ & 81 & 25.47 \\
\hline $25-34$ & 157 & 49.37 \\
\hline $35-44$ & 73 & 22.96 \\
\hline$\geq 45$ & 7 & 2.20 \\
\hline \multicolumn{3}{|l|}{ PARITY } \\
\hline 0 & 35 & 11.0 \\
\hline $1-3$ & 206 & 64.8 \\
\hline $4-6$ & 56 & 17.6 \\
\hline$>6$ & 21 & 6.6 \\
\hline \multicolumn{3}{|l|}{ Eutocic deliveries } \\
\hline 0 & 105 & 33.0 \\
\hline $1-3$ & 155 & 48.7 \\
\hline $4-6$ & 39 & 12.3 \\
\hline$>6$ & 19 & 6.0 \\
\hline \multicolumn{3}{|l|}{$\begin{array}{l}\text { Cesarean } \\
\text { deliveries }\end{array}$} \\
\hline $\begin{array}{l}0 \text { Cesarean } \\
\text { delivery }\end{array}$ & 156 & 49.1 \\
\hline $\begin{array}{l}1 \text { Cesarian } \\
\text { deliveries }\end{array}$ & 126 & 39.6 \\
\hline $\begin{array}{l}3 \text { Cesarian } \\
\text { deliveries }\end{array}$ & 9 & 2.8 \\
\hline $\begin{array}{l}>0 \mathrm{r}=4 \text { Cesarean } \\
\text { deliveries }\end{array}$ & 5 & 1.6 \\
\hline $\begin{array}{l}2 \text { Cesarian } \\
\text { deliveries } \\
\text { State of recently } \\
\text { born neonate }\end{array}$ & 22 & 6.9 \\
\hline
\end{tabular}




\begin{tabular}{lrr}
\hline Dead & 106 & 33.3 \\
& & \\
Alive & 212 & 66.7 \\
Total & 318 & 100.0 \\
\hline
\end{tabular}

Table.2 District Hospitals and their respective number of patients they referred

$\begin{array}{lrr}\text { MIBIRIZI } & 66 & 20.8 \\ \text { BUSHENGE } & 23 & 7.2 \\ \text { KIBOGORA } & 21 & 6.6 \\ \text { GIHUNDWE } & 37 & 11.6 \\ \text { KIGEME } & 45 & 14.2 \\ \text { KADUHA } & 18 & 5.7 \\ \text { MUNINI } & 20 & 6.3 \\ \text { KABUTARE } & 19 & 6.0 \\ \text { KIBIRIZI } & 17 & 5.3 \\ \text { NYANZA } & 1 & .3 \\ \text { GITWE } & 2 & .6 \\ \text { KABGAYI } & 11 & 3.5 \\ \text { RUHANGO } & 17 & 5.3 \\ \text { KILINDA } & 8 & 2.5 \\ \text { KIBUYE } & 13 & 4.1 \\ \text { Total } & 318 & 100.0\end{array}$

MIBIRIZI DH is the first DH to refer more patients 20.8\% followed by KIGEME DH. DHs from Western Province have roughly a half of transfers to CHUB during our study period (MIBIRIZI DH : $20.8 \%$, BUSHENGE DH: 7.2\%, KIBOGORA: 6.6\%, GIHUNDWE: 11.6\%).

Table.3 Reasons of referral

\begin{tabular}{lrr}
\hline REASON OF REFERENCE & Frequency & Percent \\
\hline Post-C/S PPH and stable patient & 27 & 8.5 \\
Post-NSVD PPH and Stable patient & 30 & 9.4 \\
Post-C/S PPH shock & 21 & 6.9 \\
Post-C/S PPH Shock and post-C/S delivery peritonitis & 6 & 1.9 \\
Post-NSVD PPH and severe malaria in post-partum & 1 & .3 \\
Post-NSVD PPH and anemia & 1 & .3 \\
Post-NSVD PPH Shock & 26 & 8.2 \\
Post-Cesarian delivery pelviperitonitis plus septic shock & 2 & .6 \\
Post-Cesarian delivery pelviperitonitis plus other & 1 & .3 \\
Pre-eclampsia or Eclampsia & 18 & 5.6 \\
Endometritis & 15 & 4.7 \\
Severe malaria in post-partum & 3 & .9 \\
Sever malaria in post-partum plus DVT & 1 & .3 \\
Post-partum anemia & 8 & 2.5 \\
Post-partum anemia plus other & 3 & .9 \\
Superficial wound infection & 8 & 2.5 \\
Septic shock & 2 & .6 \\
DVT & 7 & 2.2 \\
Other & 137 & 43.1 \\
Total & 318 & 100.0 \\
\hline
\end{tabular}


Table.4 Diagnosis and management at CHUB/OBGYN

\begin{tabular}{|c|c|c|c|c|c|c|c|}
\hline & & 1018 & NT A & $3 / \mathrm{OE}$ & & & \\
\hline $\begin{array}{l}\text { Diagnosis at } \\
\text { CHUB }\end{array}$ & A & B & $\mathrm{C}$ & $\mathrm{D}$ & $\mathrm{E}$ & $\mathrm{F}$ & TOTAL \\
\hline $\begin{array}{l}\text { Post-C/S PPH and } \\
\text { stable patient }\end{array}$ & 0 & 2 & 4 & 8 & 2 & 10 & 26 \\
\hline $\begin{array}{l}\text { Post-C/S PPH + Post- } \\
\text { partum anemia }\end{array}$ & 3 & 1 & 3 & 1 & 1 & 10 & 19 \\
\hline $\begin{array}{l}\text { Post-NSVD PPH and } \\
\text { stable patient and DVT }\end{array}$ & 0 & 0 & 0 & 0 & 1 & 0 & 1 \\
\hline $\begin{array}{l}\text { Post-NSVD PPH and } \\
\text { stable patient plus post- } \\
\text { partum anemia }\end{array}$ & 0 & 0 & 0 & 0 & 0 & 1 & 1 \\
\hline $\begin{array}{l}\text { Post-NSVD PPH, Stable } \\
\text { patient + Endometritis }\end{array}$ & 0 & 1 & 3 & 6 & 2 & 7 & 19 \\
\hline $\begin{array}{l}\text { Post-C/S PPH Shock } \\
\text { plus post-partum anemia }\end{array}$ & 3 & 0 & 1 & 1 & 3 & 2 & 10 \\
\hline $\begin{array}{l}\text { Post-NSVD PPH shock } \\
\text { plus other }\end{array}$ & 2 & 0 & 0 & 0 & 0 & 0 & 2 \\
\hline $\begin{array}{l}\text { Post-NSVD PPH shock } \\
+ \text { Severe malaria in } \\
\text { post-partum }\end{array}$ & 1 & 0 & 1 & 16 & 5 & 2 & 25 \\
\hline Subtotal & 9 & 4 & 12 & 32 & 14 & 32 & $\begin{array}{r}103(32.38 \\
\%)\end{array}$ \\
\hline Post-C/S Peritonitis & 0 & 0 & 0 & 5 & 3 & 1 & 9 \\
\hline $\begin{array}{l}\text { Post-C/S } \\
\text { Pelviperitonitis + } \\
\text { Endometritis }\end{array}$ & 1 & 1 & 1 & 0 & 16 & 1 & 20 \\
\hline Subtotal & 1 & $\mathbf{1}$ & 1 & 5 & 19 & 2 & $29(9.11 \%)$ \\
\hline Endometritis and other & 0 & 0 & 0 & 0 & 0 & 1 & 1 \\
\hline $\begin{array}{l}\text { Superficial wound } \\
\text { infection }\end{array}$ & 0 & 2 & 3 & 4 & 1 & 4 & 14 \\
\hline $\begin{array}{l}\text { Superficial wound } \\
\text { infection plus } \\
\text { Endometritis }\end{array}$ & 0 & 1 & 2 & 0 & 0 & 2 & 5 \\
\hline Subtotal & $\mathbf{0}$ & 3 & 5 & 4 & 1 & 7 & $20(6.28 \%)$ \\
\hline $\begin{array}{l}\text { Pre-eclampsia or } \\
\text { Eclampsia }\end{array}$ & 0 & 0 & 7 & 0 & 0 & 11 & $18(5.6 \%)$ \\
\hline DVT & 0 & 1 & 2 & 0 & 0 & 2 & $5(1.57 \%)$ \\
\hline Other & 9 & 4 & 30 & 10 & 2 & 88 & 143 \\
\hline Total & 19 & 13 & 57 & 51 & 36 & 142 & 318 \\
\hline
\end{tabular}

Legend :

A : Cytotec +oxytocin +transfusion+IV fluid+ Antibiotics

B: Cytotec +oxytocin +transfusion+IV fluid, Others

C: Cytotec +oxytocin +transfusion+IV fluid + Antibiotics, laparatomy and uterus conserved

D: Antibiotics, laparatomy, and hysterectomy

E: Antibiotics, laparatomy and uterus conserved

$\mathrm{F}$ : Others like expectant management and observation, $\mathrm{MgSO} 4$, Antihypertensive drugs 
Table.5 Health conditions at discharge day and Hospital stay

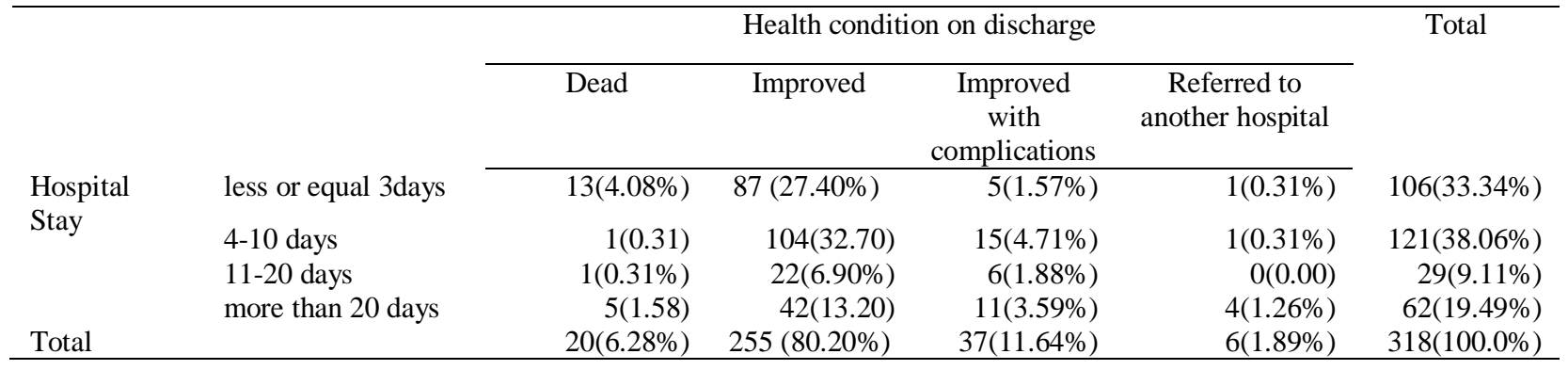

Table.6 Diagnosis at CHUB/OBGYN and complications

\begin{tabular}{|c|c|c|c|c|c|c|c|c|}
\hline & & & & & Complication & & & \\
\hline Diagnosis at CHUB & $\begin{array}{l}\text { stroke with } \\
\text { hemiplegia/ } \\
\text { hemiparesis }\end{array}$ & $\begin{array}{l}\text { ureteric or } \\
\text { bladder } \\
\text { injury }\end{array}$ & Fistula & other & $\begin{array}{l}\text { No } \\
\text { complication }\end{array}$ & $\begin{array}{l}\text { Bowel injury } \\
\text { resulted in } \\
\text { colostomy or } \\
\text { ileostomy plus } \\
\text { fistula }\end{array}$ & $\begin{array}{l}\text { Ureteric or } \\
\text { bladder } \\
\text { injury plus } \\
\text { Fistula }\end{array}$ & TOTAL \\
\hline Post-C/S PPH and stable patient & 0 & 0 & 0 & 10 & 16 & 0 & 0 & 26 \\
\hline Post-C/S PPH + Post-partum anemia & 0 & 0 & 3 & 8 & 8 & 0 & 0 & 19 \\
\hline $\begin{array}{l}\text { Post-NSVD PPH and stable patient and } \\
\text { DVT }\end{array}$ & 0 & 0 & 0 & 1 & 0 & 0 & 0 & 1 \\
\hline $\begin{array}{l}\text { Post-NSVD PPH and stable patient } \\
\text { plus post-partum anemia }\end{array}$ & 0 & 0 & 0 & 0 & 1 & 0 & 0 & 1 \\
\hline $\begin{array}{l}\text { Post-NSVD PPH, Stable patient + } \\
\text { Endometritis }\end{array}$ & 0 & 0 & 0 & 12 & 7 & 0 & 0 & 19 \\
\hline $\begin{array}{l}\text { Post-C/S PPH Shock plus post-partum } \\
\text { anemia }\end{array}$ & 0 & 0 & 3 & 2 & 5 & 0 & 0 & 10 \\
\hline Post-NSVD PPH shock plus other & 0 & 0 & 0 & 0 & 2 & 0 & 0 & 2 \\
\hline $\begin{array}{l}\text { Post-NSVD PPH shock + Severe } \\
\text { malaria in post-partum }\end{array}$ & 0 & 0 & 0 & 7 & 18 & 0 & & 25 \\
\hline Subtotal & $\mathbf{0}$ & $\mathbf{0}$ & 6 & 40 & 57 & $\mathbf{0}$ & $\mathbf{0}$ & $103(32.38 \%)$ \\
\hline Post-C/S Peritonitis & 0 & 2 & 3 & 2 & 0 & 1 & 1 & 9 \\
\hline $\begin{array}{l}\text { Post-C/S Pelviperitonitis + } \\
\text { Endometritis }\end{array}$ & 0 & 0 & 2 & 1 & 16 & 0 & 1 & 20 \\
\hline Subtotal & $\mathbf{0}$ & 2 & 5 & 3 & 16 & 1 & 2 & $29(9.11 \%)$ \\
\hline Endometritis and other & 0 & 0 & 0 & 0 & 1 & 0 & 0 & 1 \\
\hline Superficial wound infection & 0 & 1 & 0 & 4 & 9 & 0 & 0 & 14 \\
\hline $\begin{array}{l}\text { Superficial wound infection plus } \\
\text { Endometritis }\end{array}$ & 0 & 0 & 0 & 5 & 0 & 0 & 0 & 5 \\
\hline Subtotal & $\mathbf{0}$ & 1 & $\mathbf{0}$ & 9 & 10 & $\mathbf{0}$ & $\mathbf{0}$ & $20(6.28 \%)$ \\
\hline Pre-eclampsia or Eclampsia & 2 & 0 & 0 & 8 & 8 & 0 & 0 & $18(5.6 \%)$ \\
\hline DVT & 0 & 3 & 0 & 2 & 0 & 0 & 0 & $5(1.57 \%)$ \\
\hline Other & 0 & 0 & 0 & 22 & 121 & 0 & 0 & 143 \\
\hline Total & 2 & 6 & 11 & 84 & 212 & 1 & 2 & 318 \\
\hline
\end{tabular}


Table.7 Time spent at DH before transfer

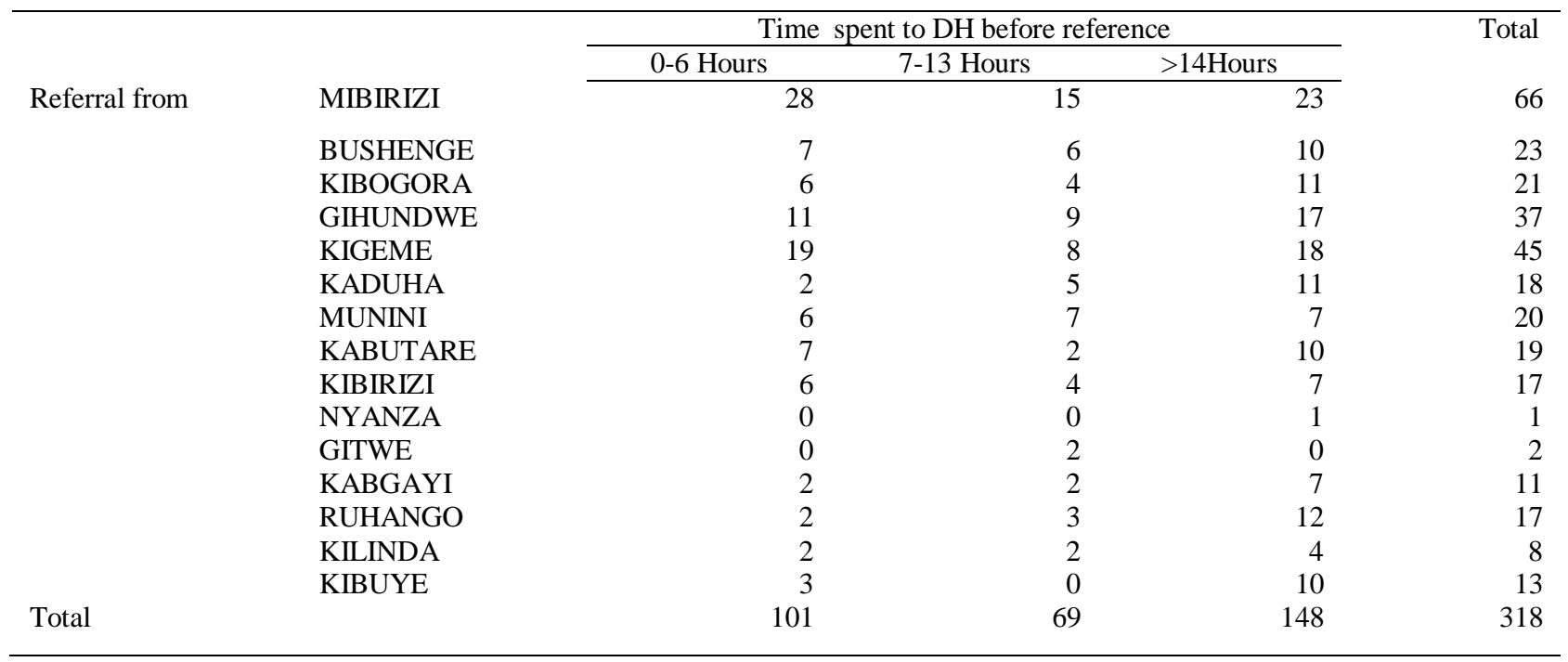

In our study period, case fatality rate was $6.3 \%(20 / 318)$. This means that $6.3 \%$ of all patients referred from DHs to CHUB/OBGYN departments died and most of them $65 \%(13 / 20)$ patients died within 3days of admission, $40 \%$ (8/20) died in ICU. They died because of the severity of their conditions at admission and probably because of the delay in transferring them or consulting as most of them $(72.3 \%)$ were managed with the right at OBGYN department from their admission.

This study revealed that some patients had various kinds of complications and depending on the initial diagnosis while majority of them were discharged with no complications. Here our attention was on long term complications and complications that interfere with the social life of the patient after discharge like fistulas.

Patients referred for $\mathrm{PPH}$ and Post-C/S peritonitis had more complications among as 44.6\% (46/103) patients had kinds fistula mainly vesico-vaginal fistulas. Among patients who were diagnosed to have post$\mathrm{C} / \mathrm{S}$ peritonitis, $17.2 \%(5 / 29)$ had fistulas also mainly enterocutaneous fistulas and
$6.8 \%(2 / 29)$ had bowel injury with colostomy or ileostomy plus fistula. Those results show how much those patients with fistula are suffering even after discharge as they can be marginalized and abandoned by their family.

This study did not compare complications among patients managed at CHUB and patients referred from district hospitals and for the best of our knowledge there is no any other similar study done at CHUB/OBGYN department. Two years-period may be enough period to have a general picture of how patients are being managed at DHs and this may serve as data base when supervisions are to be done. Training of DHs staff in emergency obstetric care and essential surgical skills and encouraging DHs to early refer their patients and to review their decision making when it is for $\mathrm{C} / \mathrm{S}$ to be done.

In conclusion, most patients who were referred from DHs to CHUB/OBGYN department in study period were having $\mathrm{PPH}$ and post-C/S peritonitis. Among them $6.3 \%$ were died and $80.2 \%$ were discharged home improved and the rest of them had long term 
complications. Long stay at $\mathrm{DH}$ before being referred is associated with adverse outcome and subsequent complications while early right diagnosis at CHUB/OBGYN department were associated with good outcome and less complications.

\section{Acknowledgement}

This study was funded by CHUB and we acknowledge the financial support from CHUB authority especially the Director General. Special thanks to the CHUB Ethics and Research Committee staff for their technical support and experts' guidance. We acknowledge all data collectors and statisticians who helped in data collection and data analysis.

\section{Competing Interests}

The authors declare that they have no competing interests.

\section{Contribution to Authorship}

HE conceived the idea, developed study protocol, participated in data collection and statistical analysis and preparation of the article.

KBJ conceived the idea, provided expert guidance, and contributed to the editing of the article.

NJ provided reviewed the protocol, gave expert guidance and contributed the preparation of the article.

BJC provided reviewed the protocol, gave expert guidance and contributed the preparation of the article.

\section{References}

Dean, T.J., Joel, G.B., et al. Disease Control Priorities in Developing Countries.
Chap 66.Referral Hospitals, 2nd ed. Washington (DC): World Bank.

Mavalankar, D.V., Rosenfield, A. 2005. Maternal Mortality in Resource-Poor Settings: Policy Barriers to Care. Am J Public Health, 95(2): 200-203.

WHO/UNICEF/UNFPA/World Bank. 2012. Trends in Maternal Mortality: 19902010. Geneva: World Health Organization.

(http://www.unfpa.org/public/home/m others/MMEstimates2012/ seen on 10/11/2013)

Ferdous, J., Ahmed, A., Dasgupta, S.K., Jahan, M., Huda, F.A., Ronsmans, C., Koblinsky, M., Chowdhury, M.E. 2012. Occurrence and Determinants of Postpartum Maternal Morbidities and Disabilities among Women in Matlab, Bangladesh. J. Health Popul. Nutr., 30(2):143-58.

Conde-Agudelo, A, Belizan, J.M., Lammers, C. 2004. Maternal-perinatal morbidity and mortality associated with adolescent pregnancy in Latin America: Cross-sectional study. American J. Obstetrics and Gynecol., 192: 342-349.

WHO. Maternal mortality. Fact sheet N³48, May 2012 (http://www.who.int/mediacentre/facts heets/fs348/en/ seen on 12/11/2013 )

National institute of statistics, Rwanda., Ministry of Finance and Economic Planning, Rwanda., Ministry of Health, Rwanda., The DHS Program ICF International Rockville,Maryland, USA., Rwanda Demographic and Health Survey 2014-2015.Final Report. March 2016.

Pembe, A.B., Carlstedt, A., Urassa, D.P., Lindmark, G., Nyström, L, Darj, E. 2010. Effectiveness of maternal referral system in a rural setting: a case study from Rufiji district, 
Tanzania. BMC Health Serv Res., 3: 10: 326.

Mamady, C., Johanne Sundby, Siri Vangen. 2009. Availability and quality of emergency obstetric care in Gambia's main referral hospital: women-users' testimonies. Reproductive Health, 6: 5.

Iyengar, K. 2012. Early Postpartum Maternal Morbidity among Rural Women of Rajasthan, India: A Community-based Study. J. Health Popul. Nutr., 30(2): 213-225. PMCID: PMC 3397332

Mantel, G.D., Buchman, E., Rees, H., Pattinson, R.C. 1998. Severe acute maternal morbidity: a pilot study of a definition for a near-miss. BJOG, 105(9): 985-990.

Shah, M., Wright, J.D. 2009. Surgical intervention in the management of post-partum haemorrhage. Semin Perinatol, 33: 109-14.

Bassey, G, et al. 2016. Emergency peripartum hysterectomy in a low resource setting: a 5-year analysis. Niger J. Med., accessed on 13april 2016.

Knight, H.E., Self, A., Kennedy, .SH. 2013. Why Are Women Dying When They Reach Hospital on Time? A Systematic Review of the 'Third Delay'. PLoS one, 21: 8(5):e63846

Mavalankar, D., Rosenfield, A. 2005. Maternal mortality in resourcepoor settings: policy barriers to care. Am. J. Public Health, 95: 200203.

WHO/UNICEF/WORLDBANK/ UNFPA. Maternal Mortality Reduction Programme in Rwanda. Geneva. World Health Organization. (http://rwanda.unfpa.org/2011/03/23/3 152/maternal_mortality_reduction_pro gramme_in_rwanda/ seen on $05 / 11 / 2013$ )

Rwanda. 2012. Health In Rwanda Is Improving! Mid Term Review (Mtr) Of The Rwanda Second Health Sector Strategic Plan (HSSP II, July 2009 June 2012). Ministry of Health, Kigali-Rwanda.

Litorp, H., Kidanto, H.L., et al. 2014. Maternal near-miss and death and their association with caesarean section complications: a cross-sectional study at a university hospital and a regional hospital in Tanzania. BMC Pregnancy Childbirth, 14: 244.

Mohammadi, S,. Essén, B., Fallahian, M., Taheripanah, R., Saleh Gargari, S., Källestål, C. 2016. Maternal near-miss at university hospitals with cesarean overuse: an incident case-control study. Acta Obstet. Gynecol. Scand.

Stephen Rulisa, Immaculee Umuzira-nenge, Maria Small and Jos van Roosmalen. 2015. Maternal near miss and mortality in a tertiary care hospital in Rwanda. BMC Pregnancy and Childbirth, $\quad$ 15: 203.

\section{How to cite this article:}

Habimana, E., J.B. Kalibushi, J. Ndoli and Bayingana, C. 2016. Morbidity and Mortality among Patients Referred from District Hospitals to Butare University Teaching Hospital Chub/Obgyn Department in Post-Partum and Post-Operative Period. Int.J.Curr.Microbiol.App.Sci. 5(7): 135-147. doi: http://dx.doi.org/10.20546/ijcmas.2016.507.013 